\title{
Simultaneous input and parameter estimation with input observers and set-membership parameter bounding: theory and an automotive application
}

\author{
I. Kolmanovsky ${ }^{1, *, \dagger}$, I. Sivergina ${ }^{2,+}$ and J. Sun ${ }^{3, \S}$ \\ ${ }^{1}$ Ford Motor Co., Powertrain Control Research and Advanced Engineering, Dearborn, MI, U.S.A. \\ ${ }^{2}$ Department of Science and Mathematics, Kettering University, Flint, MI, U.S.A. \\ ${ }^{3}$ Department of Naval Architecture and Marine Engineering, The University of Michigan, Ann Arbor, MI, U.S.A.
}

\begin{abstract}
SUMMARY
The paper addresses an on-line, simultaneous input and parameter estimation problem for a first-order system affected by measurement noise. This problem is motivated by practical applications in the area of engine control. Our approach combines an input observer for the unknown input with a set-membership algorithm to estimate the parameter. The set-membership algorithm takes advantage of a priori available information such as (i) known bounds on the unknown input, measurement noise and time rate of change of the unknown input; (ii) the form of the input observer in which the unknown parameter affects only the observer output; and (iii) the input observer error bounds for the case when the parameter is known exactly. The asymptotic properties of the algorithm as the observer gain increases are delineated. It is shown that for accurate estimation the unknown input needs to approach the known bounds a sufficient number of times (these time instants need not be known). Powertrain control applications are discussed and a simulation example based on application to engine control is reported. A generalization of the basic ideas to higher order systems is also elaborated. Copyright (C) 2006 John Wiley \& Sons, Ltd.
\end{abstract}

KEY WORDS: input observers; set-membership parameter estimation; adaptive control; engine control

\section{INTRODUCTION}

In this paper, we consider a problem of simultaneous input and parameter estimation. Although generalizations to higher order systems will be touched upon later in the paper, for the most part our developments concern a first-order system,

$$
\dot{x}=\theta^{\mathrm{T}} z(t)+u(t)
$$

In (1), $x$ is a scalar state variable, $u$ is a scalar input variable, $\theta$ is an $m$-vector of constant parameters, and $z(t)$ is an $m$-vector. Our objective is, by measuring $x(t)$ and from the assumption

\footnotetext{
${ }^{*}$ Correspondence to: I. Kolmanovsky, P.O. Box 2053, MD 2036, 2101 Village Road, Dearborn, MI 48124, U.S.A.

†E-mail: ikolmano@ford.com

†E-mail: isivergi@kettering.edu

${ }^{\S}$ E-mail: jingsun@umich.edu
} 
that $z(t)$ is known at each time $t$ (but not prior to time $t$ ), to simultaneously estimate the unknown input $u(t)$ and the unknown constant parameter vector, $\theta$.

This problem (even in the first-order case) arises quite frequently in applications. For instance, in mechanical systems first-order dependencies exist between translational velocity and forces, angular rate of rotation and torques, pressure or mass in a volume and inlet/outlet flows, etc. In these situations, the practical need to estimate and adapt models of unmeasured forces, torques or flows can rather naturally lead to the problem formulation which we treat here. We will touch upon several concrete examples of this kind, which arise in the automotive applications, later in the paper.

In the subsequent treatment of this problem, we assume that the signal $x(t)$ can be measured but its measurements are contaminated by noise, $\zeta(t)$, so that

$$
y(t)=x(t)+\zeta(t), \quad \sup _{t \geqslant 0}|\zeta(t)| \leqslant \mu
$$

where $\mu$ is a known upper bound on the noise."

It is clear that without additional assumptions $\theta$ and $u$ are not uniquely identifiable from the observed time-histories of $x$ and $z$. Indeed, if $\theta$ and $u$ are such that $\dot{x}(t)=\theta^{\mathrm{T}} z(t)+u(t)$, then with $\tilde{u}(t)=u(t)+\frac{1}{2} \theta^{\mathrm{T}} z(t), \tilde{\theta}=\frac{1}{2} \theta$ it is also true that $\dot{x}(t)=\tilde{\theta}^{\mathrm{T}} z(t)+\tilde{u}(t)$. As we will show in this paper, if the bounds on the unknown input, $u$, and its time rate of change are known and are tight at some time instants (which do not need to be known), then $\theta$ and $u$ can be accurately estimated. Specifically, let $\underline{u}(t)$ and $\bar{u}(t)$ denote, respectively, the lower and upper bounds on $u(t)$ that are known at each time $t$ (but may not be known prior to time $t$ ), and let $h$ denote the $a$ priori known bound on $|\dot{u}|$, i.e.

$$
\begin{gathered}
\underline{u}(t) \leqslant u(t) \leqslant \bar{u}(t), \quad t \geqslant 0 \\
\sup _{t \geqslant 0}|\dot{u}(t)| \leqslant h
\end{gathered}
$$

Even if bounds (3), (4) are known, additional conditions on their tightness at some time instants will be needed. Indeed, if $\underline{u}(t)<u(t)<\bar{u}(t), t \geqslant 0, \sup _{t \geqslant 0}|\dot{u}(t)|<h$ and $\dot{x}=\theta^{\mathrm{T}} z(t)+u(t)$ then for $\tilde{u}(t)=u(t)+\varepsilon z(t), \tilde{\theta}=\theta-\varepsilon$, where $|\varepsilon|$ is sufficiently small, it also follows that $\dot{x}(t)=\tilde{\theta}^{\mathrm{T}} z(t)+\tilde{u}(t), \quad \underline{u}(t) \leqslant \tilde{u}(t) \leqslant \bar{u}(t), \quad t \geqslant 0, \quad$ and $\sup _{t \geqslant 0}|\dot{\tilde{u}}(t)| \leqslant h$. Specific bound tightness conditions that guarantee accurate input and parameter estimation will be developed in this paper.

Bound tightness conditions are typically used in bounded error identification problems (see e.g. Reference [1]) to assure parameter convergence. Note, however, that system (1), (2) is not in the form treated in Reference [1]. Earlier conference papers [2,3] have described our approach and have included some of the results of this paper.

An estimation algorithm should also take an advantage of any a priori known bounds on the unknown parameter. Specifically, we assume that at $t=0$ it is known that

$$
\theta \in \Theta^{0}
$$

where $\Theta^{0}$ is a specified compact set in $\mathbf{R}^{m}$.

\footnotetext{
$\overline{{ }^{\top}}$ The functions $z$ and $\zeta$ are assumed to be Lebesgue-measurable and bounded.
} 


\section{SIMULTANEOUS INPUT AND PARAMETER ESTIMATION}

The paper is organized as follows. In Section 2, we review the estimation procedure for $u$ based on the input observer proposed in Reference [4] for the case when $\theta$ is known. In Section 3, we combine this input observer with a set-membership [5-7] algorithm to estimate $\theta$ on-line. The set-membership algorithm takes advantage of bounds (3), (4), (5) and of the input observer error bound if the parameter were accurately known. A special structure of the input observer, with the parameter affecting only the observer output equation, facilitates the application of the set-membership procedure. In Section 4, we summarize the asymptotic properties of our input and parameter estimation algorithm, as the observer gain increases and the measurement noise magnitude decreases. The computational implementation of the set-membership algorithm updates is briefly discussed in Section 5. In Section 6, we discuss the applications of the results to internal combustion engines.

For the special case of $m=1$, our approach and the relevant results have been previously reported in the conference article [2]. While our approach is closest to Reference [1], the problem can be also treated with other techniques, including References [8-11]. Reference [12] applies these techniques to a vehicle adaptive control problem in which vehicle mass and the timevarying road grade are simultaneously estimated.

Note that a higher dimensional case, $\dot{X}=Z^{\mathrm{T}}(t) \theta+u(t)$, where $X(t)$ is an $n$-vector, $u(t)$ is an $n$-vector and $Z(t)$ is an $m \times n$ matrix can be viewed as a set of $n$ systems of form (1); the bounds for $\theta$ on the basis of each of these first-order systems can be intersected to obtain tighter bounds for $\theta$. Thus this higher dimensional case reduces to the first-order case considered in the paper. More details about this approach are provided in Appendix A.

\section{THE INPUT OBSERVER}

In this section, we describe the design of an input observer for system (1), (2) in the case when $\theta$ is accurately known. Specifically, we consider an input observer in the form proposed in Reference [4],

$$
\begin{aligned}
\hat{u}(t) & =\varepsilon(t)+\alpha_{0} y(t)+\theta^{\mathrm{T}} \phi(t) \\
\dot{\phi} & =-\alpha_{0} \phi-\alpha_{0} z \\
\dot{\varepsilon} & =-\alpha_{0} \varepsilon-\alpha_{0}^{2} y
\end{aligned}
$$

Here $\alpha_{0}>0$ is the observer gain and the states of the observer are $\phi$ and $\varepsilon$. Similar filters have been previously employed in the composite adaptation literature [13].

We next study the observer properties when it is initialized according to the following procedure:

$$
\begin{aligned}
\phi(0) & =z(0) \\
\varepsilon(0) & =\frac{1}{2}(\underline{u}(0)+\bar{u}(0))-\alpha_{0} y(0)-\hat{\theta}^{\mathrm{T}} z(0)
\end{aligned}
$$

where $\hat{\theta} \in \Theta^{0}$. Note that with $\hat{\theta}=\theta$, it follows that $\hat{u}(0)=\frac{1}{2}(\underline{u}(0)+\bar{u}(0))$, which is the best estimate of $u(0)$. The subsequent developments require, however, that we also characterize the worst-case error when the observer is initialized with $\hat{\theta} \neq \theta$. The observer error bounds are obtained in the following. 


\section{Proposition 1}

If (1)-(6) hold, then for any $\phi(0), z(0)$ the error $\hat{u}(t)-u(t)$ is ultimately bounded (as $t \rightarrow \infty$ ) in an interval $\left[-2 \alpha_{0} \mu-\left(h / \alpha_{0}\right), 2 \alpha_{0} \mu+\left(h / \alpha_{0}\right)\right]$. If, in addition, (7) holds then the error between $\hat{u}(t)$ and $u(t)$ satisfies the following bound:

$$
\begin{gathered}
|\hat{u}(t)-u(t)| \leqslant R_{\alpha_{0} \mu}(t) \\
R_{\alpha_{0} \mu}(t)=\alpha_{0} \mu+\left(\mathrm{e}^{-\alpha_{0} t}\left(\frac{3}{4}(\bar{u}(0)-\underline{u}(0))^{2}+3 \alpha_{0}^{2} \mu^{2}+3 \max _{\theta, \hat{\theta} \in \Theta^{0}}\left((\theta-\hat{\theta})^{\mathrm{T}} z(0)\right)^{2}\right)+\left(\alpha_{0} \mu+\frac{h}{\alpha_{0}}\right)^{2}\right)^{1 / 2}
\end{gathered}
$$

Proof

Let

$$
\begin{aligned}
\tilde{\hat{u}} & =\varepsilon(t)+\alpha_{0} x(t)+\theta^{\mathrm{T}} \phi(t) \\
& =\hat{u}(t)-\alpha_{0} \zeta(t)
\end{aligned}
$$

and

$$
V=\frac{1}{2}(\tilde{\hat{u}}-u)^{2}
$$

In the proof, we make repeated use of the identities $(a+b+c)^{2} \leqslant 3 a^{2}+3 b^{2}+3 c^{2}, 2|a b| \leqslant$ $\left(a^{2} / d\right)+b^{2} d$ that hold for any $a, b, c$ and $d>0$. By differentiating $V$ with respect to time, we obtain

$$
\begin{aligned}
\dot{V} & =\left(-\alpha_{0} \varepsilon-\alpha_{0}^{2} x-\alpha_{0}^{2} \zeta+\alpha_{0} \theta^{\mathrm{T}} z+\alpha_{0} u+\theta^{\mathrm{T}}\left(-\alpha_{0} \phi\right)+\theta^{\mathrm{T}}\left(-\alpha_{0} z\right)-\dot{u}\right)(\tilde{\hat{u}}-u) \\
& =\left(-\alpha_{0}\left(\varepsilon+\alpha_{0} x+\theta^{\mathrm{T}} \phi-u\right)-\alpha_{0}^{2} \zeta-\dot{u}\right)(\tilde{\hat{u}}-u) \\
& =-\alpha_{0}(\tilde{\hat{u}}-u)^{2}+\left(-\alpha_{0}^{2} \zeta-\dot{u}\right)(\tilde{\hat{u}}-u) \\
& \leqslant-\frac{\alpha_{0}}{2}(\tilde{\hat{u}}-u)^{2}+\frac{1}{2 \alpha_{0}}\left(\alpha_{0}^{2} \mu+\sup _{t}|\dot{u}(t)|\right)^{2} \\
& =-\alpha_{0} V+\frac{1}{2 \alpha_{0}}\left(\alpha_{0}^{2} \mu+\sup _{t}|\dot{u}(t)|\right)^{2}
\end{aligned}
$$

Recall that if for some constant $r$,

$$
\dot{V} \leqslant-\alpha_{0} V+r
$$

then

$$
V(t) \leqslant \mathrm{e}^{-\alpha_{0} t} V(0)+\left(1-\mathrm{e}^{-\alpha_{0} t}\right) \frac{r}{\alpha_{0}} \leqslant \mathrm{e}^{-\alpha_{0} t} V(0)+\frac{r}{\alpha_{0}}
$$

Using this and (4), we obtain

$$
\begin{gathered}
V(t) \leqslant \mathrm{e}^{-\alpha_{0} t} V(0)+\frac{\left(\alpha_{0}^{2} \mu+\sup _{t}|\dot{u}(t)|\right)^{2}}{2 \alpha_{0}^{2}} \\
|\tilde{\hat{u}}(t)-u(t)| \leqslant \sqrt{\mathrm{e}^{-\alpha_{0} t}(\tilde{\hat{u}}(0)-u(0))^{2}+\frac{\left(\alpha_{0}^{2} \mu+h\right)^{2}}{\alpha_{0}^{2}}}
\end{gathered}
$$


This inequality, (2) and $\hat{u}(t)=\tilde{\hat{u}}+\alpha_{0} \zeta(t)$ show that the error $\hat{u}(t)-u(t)$ is ultimately bounded (as $t \rightarrow \infty)$ in an interval $\left[-2 \alpha_{0} \mu-\left(h / \alpha_{0}\right), 2 \alpha_{0} \mu+\left(h / \alpha_{0}\right)\right]$. To demonstrate (8), we need to upper bound $(\tilde{\hat{u}}(0)-u(0))^{2}$ under the assumption that (7) holds. From (7), we have

$$
\tilde{u}(0)=\frac{1}{2}(\underline{u}(0)+\bar{u}(0))-\alpha_{0} \zeta(0)+(\theta-\hat{\theta})^{\mathrm{T}} z(0)
$$

Using (3), we conclude that

$$
\begin{aligned}
(\tilde{\hat{u}}(0)-u(0))^{2} & \leqslant\left(\left(\frac{1}{2}(\underline{u}(0)+\bar{u}(0))-u(0)\right)-\alpha_{0} \zeta(0)+(\theta-\hat{\theta})^{\mathrm{T}} z(0)\right)^{2} \\
& \leqslant \frac{3}{4}(\bar{u}(0)-\underline{u}(0))^{2}+3 \alpha_{0}^{2} \mu^{2}+3 \max _{\theta, \hat{\theta} \in \Theta^{0}}\left((\theta-\hat{\theta})^{\mathrm{T}} z(0)\right)^{2}
\end{aligned}
$$

and (8) follows. The proof is complete.

\section{Remark 1}

According to Proposition 1, the error $\hat{u}(t)-u(t)$ is guaranteed to be ultimately bounded (as $t \rightarrow \infty)$ in an interval $\left[-2 \alpha_{0} \mu-\left(h / \alpha_{0}\right), 2 \alpha_{0} \mu+\left(h / \alpha_{0}\right)\right]$. Consequently, increasing the observer gain, in general, improves the accuracy of estimating the input but it may make the observer more sensitive to measurement noise. The interval ultimately bounding the error and $R_{\alpha_{0} \mu}(t)$ for large $t$ are minimized if $\alpha_{0}=\sqrt{h /(2 \mu)}$.

The asymptotic properties of the error bound are summarized in the following.

\section{Proposition 2}

Suppose the assumptions of Proposition 1 (including (7)) hold and $\alpha_{0} \rightarrow \infty, \mu \rightarrow 0, \alpha_{0} \mu \rightarrow 0$. Then, $R_{\alpha_{0} \mu}(t) \rightarrow 0$, and $\hat{u}(t) \rightarrow u(t) \forall t>0$.

Proposition 2 follows immediately from the error bound (8). It implies that a large observer gain (relative to the rate of change of the input) and negligible noise guarantee accurate input estimation by the observer. Note, however, that in practical applications the sampling rate would limit the observer gain even if there is no noise.

A disadvantage of the error bound (8) is that it scales proportionally to the measurement noise. The following proposition shows that the estimation error can be small even if the measurement noise is large if this noise is slowly varying (i.e. low frequency). In fact, in this case the noise magnitude, $\mu$, is irrelevant for the error bound.

\section{Proposition 3}

Suppose (1)-(6) hold and, in addition, $\dot{\zeta}$ is a Lebesgue-measurable function such that $\sup _{t}|\dot{\zeta}(t)| \leqslant \eta$. Then for any $\phi(0), z(0)$ the error $\hat{u}(t)-u(t)$ is ultimately bounded (as $\left.t \rightarrow \infty\right)$ in an interval $\left[-\eta-\left(h / \alpha_{0}\right), \eta+\left(h / \alpha_{0}\right)\right]$. If, in addition, (7) holds then the error between $\hat{u}(t)$ and $u(t)$ satisfies the following bound:

$$
\begin{gathered}
|\hat{u}(t)-u(t)| \leqslant \bar{R}_{\alpha_{0} \eta}(t) \\
\bar{R}_{\alpha_{0} \eta}(t)=\left(\mathrm{e}^{-\alpha_{0} t}\left(\frac{1}{2}(\bar{u}(0)-\underline{u}(0))^{2}+2 \max _{\theta, \hat{\theta} \in \Theta^{0}}\left((\theta-\hat{\theta})^{\mathrm{T}} z(0)\right)^{2}\right)+\left(\eta+\frac{h}{\alpha_{0}}\right)^{2}\right)^{1 / 2}
\end{gathered}
$$


Proof

The proof follows similarly to Proposition 1 with

$$
V=\frac{1}{2}(\hat{u}-u)^{2}
$$

Then,

$$
\begin{aligned}
\dot{V} & =(\hat{u}-u)\left(\alpha_{0}(u-\hat{u})+\alpha_{0} \dot{\zeta}-\dot{u}\right) \\
& =-\alpha_{0}(u-\hat{u})^{2}+(\hat{u}-u)\left(\alpha_{0} \dot{\zeta}-\dot{u}\right) \\
& \leqslant-\alpha_{0} V+\frac{\left(\alpha_{0} \eta+h\right)^{2}}{2 \alpha_{0}}
\end{aligned}
$$

Hence,

$$
V(t) \leqslant \mathrm{e}^{-\alpha_{0} t} V(0)+\frac{\left(\eta+\left(h / \alpha_{0}\right)\right)^{2}}{2}
$$

and using

$$
\hat{u}(0)=\varepsilon(0)+\alpha_{0} y(0)+\theta^{\mathrm{T}} \phi(0)=\frac{1}{2}(\underline{u}(0)+\bar{u}(0))+(\theta-\hat{\theta})^{\mathrm{T}} \phi(0)
$$

Equation (9) follows similar to the proof of Proposition 2. The proof is complete.

Bound (9) shows, in particular, that arbitrary constant measurement offsets due to sensor drifts, i.e. $y=x+\zeta$, where $\dot{\zeta}(t) \equiv 0$ can be handled by the observer irrespective of the offset magnitude.

Remark 2

According to Proposition 3, the error $\hat{u}(t)-u(t)$ is guaranteed to be ultimately bounded (as $t \rightarrow \infty)$ in an interval $\left[-\eta-\left(h / \alpha_{0}\right), \eta+\left(h / \alpha_{0}\right)\right]$. This interval and $\bar{R}_{\alpha_{0} \eta}(t)$ for large $t$ decrease as $\alpha_{0}$ increases.

The asymptotic properties of the alternative error bound can be summarized in the following.

\section{Proposition 4}

Suppose the assumptions of Proposition 3 (including (7)) hold and $\alpha_{0} \rightarrow \infty$. Then, $\bar{R}_{\alpha_{0} \eta}(t) \rightarrow \eta$ for any $t>0$. Furthermore, if $\alpha_{0} \rightarrow \infty, \eta \rightarrow 0$ then $\bar{R}_{\alpha_{0} \eta}(t) \rightarrow 0$ for $t>0$.

An additional insight into the results in Propositions 1 and 3 can be obtained if we premultiply both sides of (1) and (2) by the transfer function $\alpha_{0} s /\left(s+\alpha_{0}\right)$. Then,

$$
\frac{\alpha_{0} s}{s+\alpha_{0}} y-\frac{\alpha_{0} s}{s+\alpha_{0}} \zeta=-\theta^{\mathrm{T}} \phi+\frac{\alpha_{0}}{s+\alpha_{0}} u
$$

After algebraic manipulations, this implies

$$
\alpha_{0} y+\varepsilon+\theta^{\mathrm{T}} \phi=\frac{\alpha_{0}}{s+\alpha_{0}} u+\frac{\alpha_{0} s}{s+\alpha_{0}} \zeta
$$

Hence,

$$
u-\hat{u}=\frac{s}{s+\alpha_{0}} u-\frac{\alpha_{0} s}{s+\alpha_{0}} \zeta
$$


This easily derived expression confirms and additionally explains the nature of results in Propositions 1 and 3 .

\section{Remark 3}

In the derivations of the error bounds and throughout the paper, we assumed that the noise only affects the measurements of $x$ and that the signal $z$ is noise-free. If the noise affects $z$, similar bounds can be derived. Suppose, for example, $\xi(t) \in \Sigma$ is the noise additive to $z(t)$, where $\Sigma \subset \mathbf{R}^{m}$ is a compact set. Suppose also that the measurement noise $\zeta(t)$ in (2) can be decomposed into the sum of two terms, $\zeta(t)=\zeta^{1}(t)+\zeta^{2}(t)$, where $\sup _{t \geqslant 0}\left|\zeta^{1}(t)\right| \leqslant \mu$ and $\sup _{t \geqslant 0}$ $\left|\dot{\zeta}^{2}(t)\right| \leqslant \eta$. This assumption on the measurement noise generalizes the two cases which we previously treated separately, and it may lead to less conservative bounds. Define

$$
\kappa=\max _{\theta \in \Theta^{0}, \xi \in \Sigma}\left|\theta^{\mathrm{T}} \xi\right|
$$

Then (7) implies the following error bound:

$$
|\hat{u}(t)-u(t)| \leqslant \tilde{R}_{\alpha_{0} \mu \eta \kappa}(t)
$$

where

$$
\begin{aligned}
\tilde{R}_{\alpha_{0} \mu \eta \kappa}(t)= & \alpha_{0} \mu+\left(\mathrm{e}^{-\alpha_{0} t}\left[(\bar{u}(0)-\underline{u}(0))^{2}+4 \alpha_{0}^{2} \mu^{2}+4 \max _{\theta, \hat{\theta} \in \Theta^{0}, \xi(0) \in \Sigma}\left(\left((\theta-\hat{\theta})^{\mathrm{T}} z(0)\right)^{2}+\left((\theta-\hat{\theta})^{\mathrm{T}} \xi(0)\right)^{2}\right)\right]\right. \\
& \left.+\left[\alpha_{0} \mu+\kappa+\eta+\frac{h}{\alpha_{0}}\right]^{2}\right)^{1 / 2}
\end{aligned}
$$

This more general bound, $\tilde{R}_{\alpha_{0} \mu \eta \kappa}(t)$, can be used in the subsequent developments in exactly the same manner as either $R_{\alpha_{0} \mu}(t)$ or $\bar{R}_{\alpha_{0} \eta}(t)$.

\section{Remark 4}

Our formulation of the input observer (6) and the derivations of the above error bounds have been performed in continuous-time. In the actual implementation of (6), inadequate (i.e. slow) sampling and approximation of (6) by a discrete-time system, such as

$$
\begin{gathered}
\hat{u}(t)=\varepsilon_{d}(t)+\alpha_{0} y(t)+\theta^{\mathrm{T}} \phi_{d}(t) \\
\phi_{d}(t+\Delta t)=\mathrm{e}^{-\alpha_{0} \Delta t} \phi_{d}(t)-\left(1-\mathrm{e}^{-\alpha_{0} \Delta t}\right) z(t) \\
\varepsilon_{d}(t+\Delta t)=\mathrm{e}^{-\alpha_{0} \Delta t} \varepsilon_{d}(t)-\left(1-\mathrm{e}^{-\alpha_{0} \Delta t}\right) \alpha_{0} y(t)
\end{gathered}
$$

may result in additional and nonnegligible errors. These errors due to sampling (easily shown to be in the order of the sampling period, $\Delta t$, under appropriate assumptions) can be estimated and included in the input observer error bounds. Their treatment and the issues of optimally selecting the sampling rate, to balance good input observer accuracy with the containable computing effort, will be addressed in the future work.

We next consider the case when both $u$ and $\theta$ are unknown. 


\section{ON-LINE PARAMETER ESTIMATION}

If the parameter $\theta$ is not known, then in the input observer (6) we replace $\theta$ by its estimate $\hat{\theta}$ so that

$$
\begin{aligned}
\hat{u}(t) & =\varepsilon(t)+\alpha_{0} y(t)+\hat{\theta}^{\mathrm{T}} \phi(t) \\
\dot{\phi} & =-\alpha_{0} \phi-\alpha_{0} z \\
\dot{\varepsilon} & =-\alpha_{0} \varepsilon-\alpha_{0}^{2} y
\end{aligned}
$$

Since $\hat{\theta}$ affects only the observer output, $\hat{u}$, and not the observer dynamics (i.e. the equations for $\dot{\phi}$ and $\dot{\varepsilon}$ ), we may view (10) as an infinite family of concurrent observers for $\hat{u}$, each corresponding to a particular value of $\hat{\theta}$.

The available information, besides (3) and (5), is either $|\zeta(t)| \leqslant \mu$ or $|\dot{\zeta}(t)| \leqslant \eta$. Suppose, for example, that $|\zeta(t)| \leqslant \mu$ and the conditions of Proposition 1 hold (the case when $|\dot{\zeta}(t)| \leqslant \eta$ and the conditions of Proposition 3 hold can be treated in the same way). Then, if in (10) we had $\hat{\theta}=\theta$ (i.e. we guessed the parameter right), we would have

$$
u(t)-R_{\alpha_{0} \mu}(t) \leqslant \hat{u}(t) \leqslant u(t)+R_{\alpha_{0} \mu}(t)
$$

Using the a priori bound (3), we obtain

$$
\underline{u}(t)-R_{\alpha_{0} \mu}(t) \leqslant \hat{u}(t) \leqslant \bar{u}(t)+R_{\alpha_{0} \mu}(t)
$$

Due to (10), inequality (11) is equivalent to

$$
\underline{u}(t)-R_{\alpha_{0} \mu}(t)-\varepsilon(t)-\alpha_{0} y(t) \leqslant \hat{\theta}^{\mathrm{T}} \phi(t) \leqslant \bar{u}(t)+R_{\alpha_{0} \mu}(t)-\varepsilon(t)-\alpha_{0} y(t)
$$

\section{Definition}

A set $\Theta(t)$ of plausible parameter estimates at time $t>0$ is the set of all $\hat{\theta} \in \mathbf{R}^{m}$ yielding (12).

Note that $\Theta(t)$ is nonempty for each $t>0$ because $\theta \in \Theta(t)$. Setting $\Theta(0)=\Theta^{0}$, we denote

$$
F(t) \triangleq \bigcap_{0 \leqslant \tau \leqslant t} \Theta(\tau)
$$

Since $\theta \in \Theta(t)$ for $t \geqslant 0, \theta \in F(t)$.

\section{Remark 5}

Suppose the time-rate of change of the noise is bounded by $\eta$ but the upper bound on the noise magnitude is unknown, as in Proposition 3. Then, (12), (13) still hold but in (12), $R_{\alpha_{0} \mu}(t)$ should be replaced by $\bar{R}_{\alpha_{0} \eta}(t)$, defined in (9).

Note that, in general, $\hat{\theta} \in F(t)$ does not uniquely identify $\hat{\theta}$. The selection of a specific $\hat{\theta} \in F(t)$ at time $t$ can be made in a number of different ways. One choice, motivated by the setmembership estimation literature (see e.g. Reference [14]), is to use the Chebyshev centre of $F(t)$. This is the point for which the maximum distance to any other point within $F(t)$ is minimized.

Since $F\left(t_{1}\right) \subset F\left(t_{2}\right)$ for $t_{1} \geqslant t_{2}, F(t)$ provides tighter and tighter overbounds of the unknown parameter $\{\theta\}$ as $t$ increases. We next seek conditions guaranteeing that $F(t)$ for 
$t>0$ provides as tight overbound of $\theta$ as possible and converges to $\{\theta\}$ as the observer gain, $\alpha_{0}$, increases.

\section{ASYMPTOTIC PROPERTIES}

Consider the asymptotic properties of the set $F(t)$, defined by (13), as $\alpha_{0} \rightarrow \infty, \mu \rightarrow 0, \mu \alpha_{0} \rightarrow 0$. Subtracting $\theta^{\mathrm{T}} \phi(t)$ from each part of inequality (12), we obtain an equivalent inequality,

$$
\begin{aligned}
& (\underline{u}(t)-u(t))+\left(u(t)-\varepsilon(t)-\alpha_{0} y(t)-\theta^{\mathrm{T}} \phi(t)\right)-R_{\alpha_{0} \mu}(t) \\
& \quad \leqslant(\hat{\theta}-\theta)^{\mathrm{T}} \phi(t) \leqslant(\bar{u}(t)-u(t))+\left(u(t)-\varepsilon(t)-\alpha_{0} y(t)-\theta^{\mathrm{T}} \phi(t)\right)+R_{\alpha_{0} \mu}(t)
\end{aligned}
$$

Applying Proposition 1, we obtain the estimate

$$
(\underline{u}(t)-u(t))-2 R_{\alpha_{0} \mu}(t) \leqslant(\hat{\theta}-\theta)^{\mathrm{T}} \phi(t) \leqslant(\bar{u}(t)-u(t))+2 R_{\alpha_{0} \mu}(t)
$$

Inequality (14) leads to the tightest constraints on $(\hat{\theta}-\theta)$ at the time instants when the bounds $\underline{u}(t)$ and $\bar{u}(t)$ are tight. Specifically, suppose there exist time instants $t_{1}^{1}, \ldots, t_{1}^{n_{1}}$ and $t_{2}^{1}, \ldots, t_{2}^{n_{2}}$ such that $u\left(t_{1}^{i}\right)=\underline{u}\left(t_{1}^{i}\right), i=1, \ldots, n_{1}, u\left(t_{2}^{i}\right)=\bar{u}\left(t_{2}^{i}\right), i=1, \ldots, n_{2}$. At these time instants, (14) implies

$$
\begin{aligned}
& \phi^{\mathrm{T}}\left(t_{1}^{i}\right)(\hat{\theta}-\theta) \geqslant-2 R_{\alpha_{0} \mu}\left(t_{1}^{i}\right), \quad i=1, \ldots, n_{1} \\
& \phi^{\mathrm{T}}\left(t_{2}^{j}\right)(\hat{\theta}-\theta) \leqslant 2 R_{\alpha_{0} \mu}\left(t_{2}^{j}\right), \quad j=1, \ldots, n_{2}
\end{aligned}
$$

Theorem 1

Suppose $F(t)$ is defined by (13) and there exist time instants $t_{1}^{1}, \ldots, t_{1}^{n_{1}}>0$ and $t_{2}^{1}, \ldots, t_{2}^{n_{2}}>0$ such that

$$
\begin{array}{ll}
u\left(t_{1}^{i}\right)=\underline{u}\left(t_{1}^{i}\right), & i=1, \ldots, n_{1} \\
u\left(t_{2}^{j}\right)=\bar{u}\left(t_{2}^{j}\right), & j=1, \ldots, n_{2}
\end{array}
$$

Define a matrix

$$
S=\left[\begin{array}{c}
z^{\mathrm{T}}\left(t_{1}^{1}\right) \\
z^{\mathrm{T}}\left(t_{1}^{2}\right) \\
\vdots \\
z^{\mathrm{T}}\left(t_{1}^{n_{1}}\right) \\
-z^{\mathrm{T}}\left(t_{2}^{1}\right) \\
\vdots \\
-z^{\mathrm{T}}\left(t_{2}^{n_{2}}\right)
\end{array}\right] \in \mathbf{R}^{\left(n_{1}+n_{2}\right) \times m}
$$

Suppose the only solution to the system of linear inequalities

$$
S \tilde{\theta} \leqslant 0, \quad \tilde{\theta} \in \mathbf{R}^{m}
$$


is $\tilde{\theta}=0$. Suppose further that $z$ in (1) is differentiable with $\dot{z}$ being bounded. Then for $t>\max \left\{t_{1}^{1}, \ldots, t_{1}^{n_{1}}, t_{2}^{1}, \ldots, t_{2}^{n_{2}}\right\}$ it follows that $F(t) \rightarrow\{\theta\}$ and $\hat{u}(t) \rightarrow u(t)$ as $\alpha_{0} \rightarrow \infty, \mu \rightarrow 0$, and $\alpha_{0} \mu \rightarrow 0$.

Proof

From (15), the following set inclusion holds for $t>\max \left\{t_{1}^{1}, \ldots, t_{1}^{n_{1}}, t_{2}^{1}, \ldots, t_{2}^{n_{2}}\right\}$ :

$$
F(t) \subset\{\theta\}+Q^{1} \bigcup Q^{2}\left(\alpha_{0}, \mu\right)
$$

where

$$
\begin{aligned}
& Q^{1}=\left\{\tilde{\theta} \in \mathbf{R}^{m}: \exists \hat{\theta} \in \Theta^{0}, \theta \in \Theta^{0} \text { such that } \tilde{\theta}=\hat{\theta}-\theta\right. \\
& \left.z^{\mathrm{T}}\left(t_{1}^{i}\right) \tilde{\theta} \leqslant 0, \quad-z\left(t_{2}^{j}\right)^{\mathrm{T}} \tilde{\theta} \leqslant 0, i=1, \ldots, n_{1}, j=1, \ldots, n_{2}\right\} \\
& \begin{aligned}
Q^{2}\left(\alpha_{0}, \mu\right)= & \left\{\tilde{\theta} \in \mathbf{R}^{m}: \exists \hat{\theta} \in \Theta^{0}, \theta \in \Theta^{0}\right. \text { such that } \\
& \tilde{\theta}=\hat{\theta}-\theta, 0<z^{\mathrm{T}}\left(t_{1}^{i}\right) \tilde{\theta} \leqslant 2 R_{\alpha_{0} \mu}\left(t_{1}^{i}\right)-\left(\phi\left(t_{1}^{i}\right)+z\left(t_{1}^{i}\right)\right)^{\mathrm{T}} \tilde{\theta} \\
& 0<-z^{\mathrm{T}}\left(t_{2}^{j}\right) \tilde{\theta} \leqslant 2 R_{\alpha_{0} \mu}\left(t_{2}^{j}\right)-\left(\phi\left(t_{2}^{j}\right)+z\left(t_{2}^{j}\right)\right)^{\mathrm{T}} \tilde{\theta} \\
& \left.i=1, \ldots, n_{1}, j=1, \ldots, n_{2} .\right\}
\end{aligned}
\end{aligned}
$$

Since $S \tilde{\theta} \leqslant 0$ implies $\tilde{\theta}=0$, it follows that $Q^{1}=\{0\}$ and $S$ has full column rank. The latter and the compactness of $\Theta^{0}$ imply that $Q^{2}\left(\alpha_{0}, \mu\right)$ is bounded. As $\alpha_{0} \rightarrow \infty$, it follows, with the help of integration by parts, that

$$
\begin{aligned}
\phi\left(t_{1}^{i}\right)+z\left(t_{1}^{i}\right) & =\mathrm{e}^{-\alpha_{0} t_{1}^{i}} z(0)-\int_{0}^{t_{1}^{i}} \mathrm{e}^{-\alpha_{0}\left(t_{1}^{i}-\tau\right)} \alpha_{0} z(\tau) \mathrm{d} \tau+z\left(t_{1}^{i}\right) \\
& =\mathrm{e}^{-\alpha_{0} t_{1}^{i}} z(0)-z\left(t_{1}^{i}\right)+\int_{0}^{t_{1}^{i}} \mathrm{e}^{-\alpha_{0}\left(t_{1}^{i}-\tau\right)} \dot{z}(\tau) \mathrm{d} \tau+z\left(t_{1}^{i}\right) \\
& \rightarrow 0
\end{aligned}
$$

for $i=1, \ldots, n_{1}$. Similarly, as $\alpha_{0} \rightarrow \infty$, it follows that $\phi\left(t_{2}^{j}\right)+z\left(t_{2}^{j}\right) \rightarrow 0, j=1, \ldots, n_{2}$. Further, as $\alpha_{0} \rightarrow \infty, \mu \rightarrow 0, \quad \alpha_{0} \mu \rightarrow 0$ it follows that $R_{\alpha_{0} \mu}\left(t_{1}^{i}\right) \rightarrow 0$ and $R_{\alpha_{0} \mu}\left(t_{2}^{j}\right) \rightarrow 0, \quad i=1, \ldots, n_{1}$, $j=1, \ldots, n_{2}$. Thus $Q^{2}\left(\alpha_{0}, \mu\right) \rightarrow\{0\}$. This completes the proof.

\section{Remark 6}

Suppose that the time-rate of change of the noise is bounded by $\eta$, as in Proposition 3 . Then, with $F(t)$ defined by (12), (13), where $R_{\alpha_{0} \mu}$ is replaced by $\bar{R}_{\alpha_{0} \eta}$ in (12), and under the remaining assumptions of Theorem 1, the result of Theorem 1 holds as $\alpha_{0} \rightarrow \infty, \eta \rightarrow 0$.

\section{Remark 7}

Theorem 1 indicates that accurate input and parameter estimation becomes possible when the unknown input touches its bounds a sufficient number of times. Note that the knowledge of what these time instants are is not required.

Figure 1 illustrates graphically conditions (17)-(18) in the case $m=2, n_{1}=2, n_{2}=1$. As Figure 1(a) shows, it may be possible to accurately estimate (in the sense of Theorem 1) 


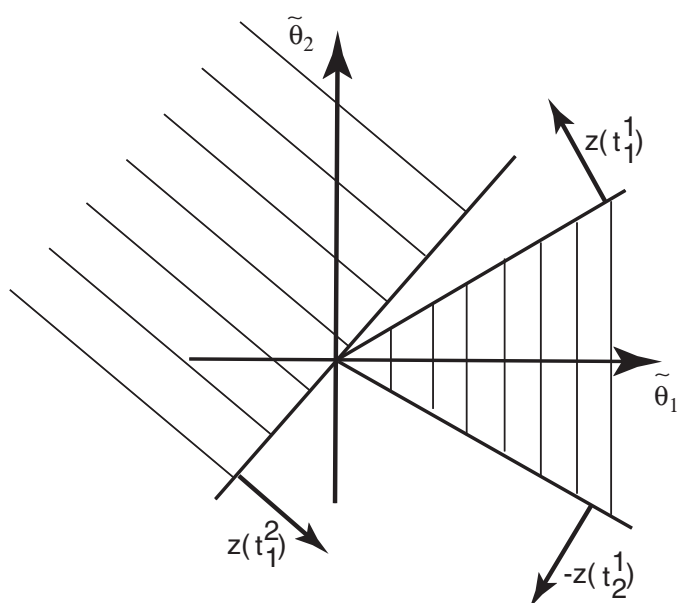

(a)

Figure 1. Graphical illustrations of conditions (17)-(18) for $m=2, n_{1}=2$ and $n_{2}=1$ : (a) conditions hold; and (b) conditions do not hold.

two unknown parameters if the unknown input touches its bounds just three times. Figure 1(b) indicates, however, that three touches may not always be enough.

In the general case, the inequality consequence, $S \tilde{\theta} \leqslant 0$ implies $\tilde{\theta}=0$, can be checked using a linear programming solver (this is a feasibility problem) or, more elegantly, via the Farkas lemma. Specifically, the necessary and sufficient condition for it to hold is the existence of a matrix $X \in \mathbf{R}^{\left(n_{1}+n_{2}\right) \times 2 m}$ with all nonnegative elements such that

$$
S^{\mathrm{T}} X=\left[\begin{array}{ccccccc}
1 & -1 & 0 & 0 & \cdots & 0 & 0 \\
0 & 0 & 1 & -1 & \cdots & 0 & 0 \\
\vdots & \vdots & \vdots & \vdots & \cdots & \vdots & \vdots \\
0 & 0 & 0 & 0 & \cdots & 1 & -1
\end{array}\right]
$$

The MATLAB $\|$ function lsqnonneg can be used for a straightforward computational check if $X$ satisfying (20) with all nonnegative elements exist.

We note that a condition from the identification literature, that the matrix $J=$ $\sum_{i=1}^{n_{1}} z\left(t_{1}^{i}\right) z^{\mathrm{T}}\left(t_{1}^{i}\right)+\sum_{j=1}^{n_{2}} z\left(t_{2}^{j}\right) z^{\mathrm{T}}\left(t_{2}^{j}\right)$ is positive-definite, is only necessary but, as simple examples in the case $m=1$ show, not sufficient for $S \tilde{\theta} \leqslant 0$ to imply $\tilde{\theta}=0$. However, if $F(t)$ is defined by (13), $J$ is positive-definite and $n_{1}=n_{2}, t_{1}^{i}=t_{2}^{i}, i=1, \ldots, n_{1}$ (i.e. $\left.u\left(t_{1}^{i}\right)=u\left(t_{1}^{i}\right)=\bar{u}\left(t_{1}^{i}\right)\right)$, then $F(t) \rightarrow\{\theta\}$ and $\hat{u}(t) \rightarrow u(t)$ for $t \geqslant \max \left\{t_{1}^{1}, \ldots, t_{1}^{n_{1}}\right\}$ as $\alpha_{0} \rightarrow \infty, \mu \rightarrow 0, \alpha_{0} \mu \rightarrow 0$. The same result holds if $F(t)$ is defined as in Remark 7 and $\alpha_{0} \rightarrow \infty, \eta \rightarrow 0$.

Suppose now that for any $T>0$ there exist time instants $t_{1}^{i}>T, i=1, \ldots, n_{1}$, and $t_{2}^{j}>T$, $j=1, \ldots, n_{2}$, which satisfy the conditions of Theorem 1 (i.e. conditions of Theorem 1 hold

\footnotetext{
${ }^{\|}$MATLAB is a registered trademark of the MathWorks Inc. of Natick, MA.
} 
persistently). Then the asymptotic bounds on $F(t)$ as $t \rightarrow \infty$ can be characterized on the basis of (19). In particular, for any $\delta>0$, there exist $T>0$ such that if the matrix $\Phi$ is defined by (17) with $z$ replaced by $\phi$ and is of full column rank, and if $t>\max \left\{t_{1}^{i}, t_{2}^{j}, i=1, \ldots, n_{1}, j=1, \ldots, n_{2}\right\}$, then

$$
F(t) \subset\{\theta\}+\left(\delta+4 \alpha_{0} \mu+\left(2 h / \alpha_{0}\right)\right)\left\|\left(\Phi^{\mathrm{T}} \Phi\right)^{-1} \Phi^{\mathrm{T}}\right\| \mathscr{B}_{\infty}
$$

where $\|\cdot\|$ denotes 1-matrix norm and $B_{\infty}$ is the $\infty$-norm unit ball in $\mathbf{R}^{m}$.

\section{COMPUTATIONAL DETAILS}

While the on-line implementation of the input observer (10) is straightforward, the on-line implementation of (13) warrants further discussion.

Firstly, the continuous-time version of (13) can be replaced by a sampled-data version, $F(t) \approx F_{d}(k)$ :

$$
\begin{aligned}
& F_{d}(k)=\bigcap_{t=0, \Delta, \ldots, k \cdot \Delta} \Theta(t), \quad k \cdot \Delta \leqslant t<(k+1) \cdot \Delta \\
& \Theta(0)=\Theta^{0}
\end{aligned}
$$

where $\Delta$ is the time period between two updates of $F_{d}$. In a recursive form,

$$
F_{d}(k+1)=F_{d}(k) \bigcap \Theta((k+1) \cdot \Delta), \quad F_{d}(0)=\Theta^{0}
$$

Note that $F(t) \in F_{d}(k)$ for $k \cdot \Delta \leqslant t<(k+1) \cdot \Delta$. Furthermore, if the assumptions of Theorem 1 (or Remark 6) hold for $t_{1}^{i}=k_{1}^{i} \Delta, t_{2}^{j}=k_{2}^{j} \Delta, k_{1}^{i}, k_{2}^{j} \in \mathbf{Z}^{+}, i=1, \ldots, n_{1}, j=1, \ldots, n_{2}$ then the conclusion of Theorem 1 (or Remark 6 , respectively) holds for $F_{d}(k), k \geqslant \max \left\{k_{1}^{1}, \ldots, k_{1}^{n_{1}}\right.$, $\left.k_{2}^{1}, \ldots, k_{2}^{n_{2}}\right\}$.

Secondly, for real-time implementation it is highly desirable that the representation of $F_{d}(k)$, update (22) and the computation of the Chebyshev centre of $F_{d}(k)$ (since $\hat{\theta}(t)$ for $k \cdot \Delta \leqslant t<(k+1) \Delta$ is set equal to the Chebyshev centre of $\left.F_{d}(k)\right)$ are as simple as possible.

Standard algorithms in the set-membership literature can be used for on-line construction of easily computable overbounds of $F_{d}(k)$. Well-developed procedures exist based on bounding methods for an intersection of an ellipsoid and a strip [15] or a parallelotope and a strip [16]. See also Reference [1].

In our work, motivated by specific automotive applications, we followed a similar approach where we even further sacrificed the tightness of the overbound for the simplicity of implementation and computations, which are important considerations for the automotive applications where computational resources (chronometrics and memory) are very limited.

Specifically, in our approach we replace the updates of $F_{d}(k)$ with the updates of an $m$-dimensional rectangle, $P(k)$, which overbounds $F_{d}(k)$, i.e. $F_{d}(k) \subset P(k)$. In the case $m=1$ a simple algorithm of [2] is sufficient. These computational procedures are reviewed in more detail in Appendix B. 


\section{POWERTRAIN CONTROL APPLICATIONS}

The first example is based on an internal combustion engine intake manifold filling and emptying dynamics,

$$
\dot{p}=\frac{R \cdot T_{m}}{V_{m}}\left(W_{\text {th }}+W_{\text {egr }}-W_{\text {cyl }}\right)
$$

where $p$ is the measured intake manifold pressure, $W_{\text {th }}$ is the flow through the engine throttle measured by a mass flow sensor, $W_{\text {egr }}$ is the flow through the exhaust gas recirculation (EGR) valve and $W_{\text {cyl }}$ is the mean-value of the flow into the engine cylinders. The flows $W_{\text {egr }}$ and $W_{\text {cyl }}$ are not measured. The $R, V_{m}$ and $T_{m}$ are, respectively, the gas constant, the intake manifold volume, and the gas temperature in the intake manifold. See Figure 2.

Both $W_{\text {cyl }}$ and $W_{\text {egr }}$ need to be accurately estimated for good air-fuel ratio and spark control, and this estimation is therefore essential for vehicle drivability, fuel economy and emissions reduction. Furthermore, an EGR flow estimate can be used for a feedforward compensation in partial air pressure control system [17]. The cylinder flow, $W_{\text {cyl }}$, can be estimated according to the following equation [18]:

$$
W_{\mathrm{cyl}}=W_{\mathrm{cyl}, 0} \triangleq \frac{N}{R T_{m}}\left(k_{\mathrm{cyl}, 0}+k_{\mathrm{cyl}, 1} \cdot p\right)
$$

where $N$ is the engine speed and $k_{\mathrm{cyl}, 0}, k_{\mathrm{cyl}, 1}$ are parameters. With aging, soot deposits may form in the intake runners (this is especially a problem in direct injection spark ignition engines, see Reference [18]). These deposits can reduce the flow area in ways dependent on unique driving and environmental factors for each vehicle, and difficult to predict in advance. The part-to-part manufacturing variabilities can also render estimate (24) inaccurate. Thus there is a benefit to using on-line adaptation to improve the cylinder flow estimate over time. For example, an adaptive model may have the form,

$$
W_{\text {cyl }}=\theta_{1} \cdot W_{\text {cyl }, 0}
$$

where $\theta_{1}$ is a constant parameter to be estimated on-line. The model for the EGR flow, $W_{\text {egr }}$, can be developed on the basis of the compressible flow 'orifice' equation,

$$
W_{\text {egr }}=f_{\text {egr }}\left(\frac{p}{p_{\text {ex }}}\right) \cdot A_{\text {egr }} \cdot \frac{p_{\text {ex }}}{\sqrt{T_{\text {ex }}}}
$$

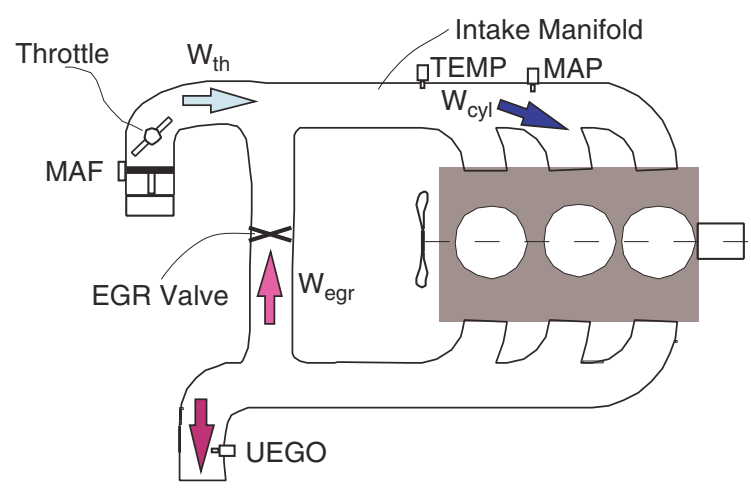

Figure 2. Internal combustion engine with exhaust gas recirculation (EGR). 
where $p_{\mathrm{ex}}$ is the exhaust pressure, $T_{\mathrm{ex}}$ is the exhaust temperature, $A_{\mathrm{egr}}$ is the effective flow area of the EGR valve which is a function of measured EGR valve position, and $f_{\text {egr }}$ is a known function of the pressure ratio across the EGR valve:

$$
f_{\text {egr }}(x)= \begin{cases}\gamma^{1 / 2}\left(\frac{2}{\gamma+1}\right)^{(\gamma+1) / 2(\gamma-1)} & \text { if } x \leqslant 0.5 \\ x^{1 / \gamma}\left\{\frac{2 \gamma}{\gamma-1}\left[1-x^{(\gamma-1) / \gamma}\right]\right\}^{1 / 2} & \text { if } x>0.5\end{cases}
$$

Here $\gamma$, the ratio of specific heats, is considered to be constant $(\gamma=1.4)$. In reality, $W_{\text {egr }}$ is difficult to estimate based on (26) because $p_{\text {ex }}$ and $T_{\text {ex }}$ are not measured, and $A_{\text {egr }}$ may change over time due to soot deposits [18]. Additionally, (26) only applies for small openings of the EGR valve, and for large openings $A_{\text {egr }}$ exhibits engine speed dependence. Thus a natural approach is to treat $W_{\text {egr }}$ as an unknown input and estimate it on-line simultaneously with $\theta_{1}$ in (25). An adaptive multiplier, $\theta_{2}$, can also be introduced to compensate mass air flow sensor drifts so that $W_{\mathrm{th}}=\theta_{2} \cdot W_{\mathrm{th}, 0}$. Specifically, if we let

$$
\begin{gathered}
x=p, \quad z_{1}=-\frac{R T_{m}}{V_{m}} W_{\mathrm{cyl}, 0}, \quad z_{2}=\frac{R T_{m}}{V_{m}} W_{\mathrm{th}, 0} \\
u=\frac{R T_{m}}{V_{m}} W_{\mathrm{egr}}, \quad \theta=\left[\begin{array}{l}
\theta_{1} \\
\theta_{2}
\end{array}\right], \quad z=\left[\begin{array}{l}
z_{1} \\
z_{2}
\end{array}\right]
\end{gathered}
$$

then the system takes the standard form (1).

We assume that in (5),

$$
\Theta^{0}=[0.7,1.9] \times[0.9,1.7]
$$

so that the nominal parameter estimates are $\hat{\theta}^{1}=\hat{\theta}^{2}=1.3$. Note that this problem is challenging since all three flows $W_{\text {cyl }}, W_{\text {th }}$ and $W_{\text {egr }}$ are uncertain and the measurements of pressure, $p$, are noisy. Our estimation algorithm will be applied to trajectories of the pressure and of the three flows resulting from changing throttle and the EGR valve positions during a $12 \mathrm{~s}$ time period. These trajectories are shown in Figure 3.

The a priori knowledge of bounds on the unknown input is not an unreasonable assumption. For example, in the EGR flow estimation problem, while (26) cannot be used to accurately estimate $W_{\text {egr }}$ it still can be used to accurately estimate the upper and lower bounds on $W_{\text {egr }}$.

The bounds on the EGR flow, $\underline{W}_{\text {egr }}$ and $\bar{W}_{\text {egr }}$, are also shown in Figure 3 . These bounds are estimated on-line from Equation (26) using measured intake manifold pressure, $p$, and known ranges of exhaust pressure, $p_{\text {ex }}$, temperature, $T_{\text {ex }}$, and EGR valve effective flow area, $A_{\text {egr }}$. In (3), $\underline{u}=\left(R T_{m} / V_{m}\right) \underline{W}_{\text {egr }}, \bar{u}=\left(R T_{m} / V_{m}\right) \bar{W}_{\text {egr }}$. The nominal cylinder flow estimate, $\hat{W}_{\text {cyl }}=\hat{\theta}_{1} W_{\text {cyl }, 0}$ with $\hat{\theta}_{1}=1.3$ is significantly higher than the actual cylinder flow, $W_{\text {cyl }}$, because the latter corresponds to $W_{\text {cyl }}=\theta_{1} W_{\text {cyl }, 0}$ with $\theta_{1}=0.8$. Similarly, the nominal throttle flow estimate, $\hat{W}_{\text {th }}=\hat{\theta}_{2} W_{\text {th, } 0}$ with $\hat{\theta}_{2}=1.3$ is significantly higher than the actual throttle flow, $W_{\text {th }}=\theta_{2} W_{\text {th }, 0}$ with $\theta_{2}=1.0$.

The upper bound on the measurement noise in (2) was assumed to be $\mu=1 \mathrm{kPa}$, and $h=101.87$ in (4) was estimated by model simulation. The input observer gain $\alpha_{0}$ was set based on Remark 1 as $\alpha_{0}=(h /(2 \mu))^{0.5}=7.14$. The time-dependent input observer error bound, 


\section{SIMULTANEOUS INPUT AND PARAMETER ESTIMATION}
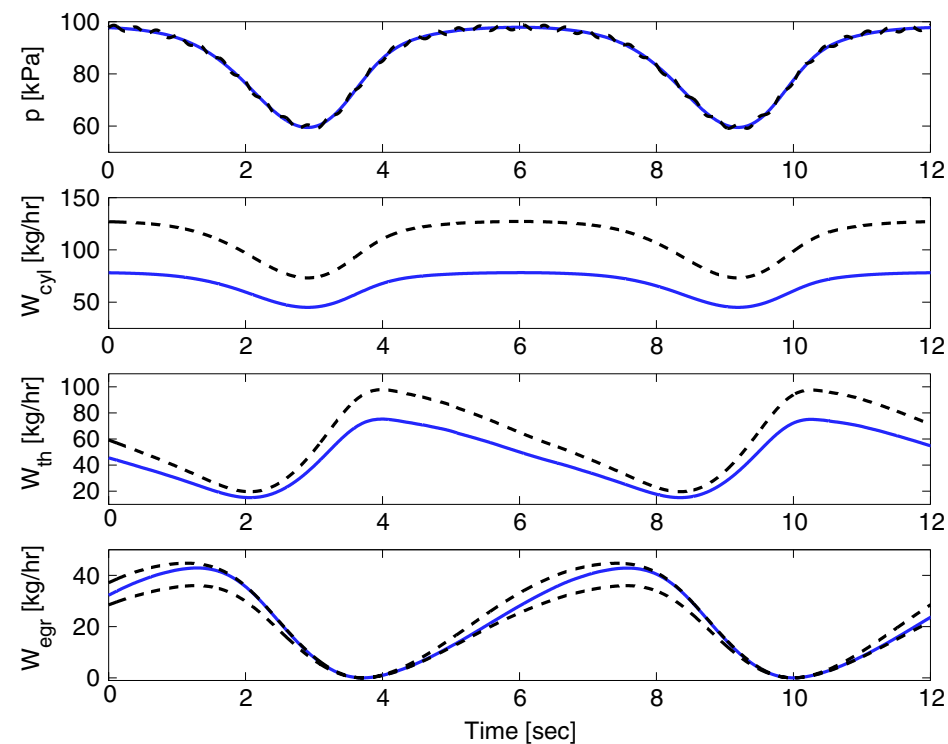

Figure 3. Time histories (from (a) at the top to (d) at the bottom) of: (a) $x=p$ (solid) and noisy measurement $y$ (dashed); (b) $W_{\text {cyl }}$ (solid) and $\hat{W}_{\text {cyl }}=\hat{\theta}_{1} W_{\text {cyl, } 0}$ with $\hat{\theta}_{1}=1.3$ (dashed); (c) $W_{\text {th }}$ (solid) and $\hat{W}_{\text {th }}=\hat{\theta}_{2} W_{\text {th, } 0}$ with $\hat{\theta}_{1}=1.3$ (dashed); and (d) $W_{\text {egr }}$ (solid), $\underline{W}_{\text {egr }}$ (dashed) and $\bar{W}_{\text {egr }}$ (dashed).

$R_{\alpha_{0} \mu}(t)$, was calculated according to (8). Figure 4 shows the time-dependent input observer error bound, $R_{\alpha_{0} \mu}(t)$, calculated according to (8).

Figure 5 shows that the simultaneous input and parameter estimation can be accomplished quite well by our algorithm. The estimated EGR flow, $\hat{W}_{\text {egr }}$, converges to the actual EGR flow, $W_{\text {egr }}$, very fast and, in fact, as Figure 6 shows, the area of the rectangle $P(k)$, which bounds plausible parameter estimates at $t=k \Delta$, is reduced by a factor of 13.5 within just $12 \mathrm{~s}$. The parameter estimates, $\hat{\theta}_{1}, \hat{\theta}_{2}$ converge to a small neighbourhood of $\theta_{1}, \theta_{2}$ within the same time period.

Other powertrain control applications include the speed dynamics of an internal combustion engine which are described by the following equation:

$$
J \dot{\omega}=\tau_{\text {eng }}-\tau_{\text {load }}
$$

where $J$ is a known inertia of the crankshaft and $\tau_{\text {load }}$ is an unknown load torque on the crankshaft. The engine torque, $\tau_{\text {eng }}$, may be nominally characterized by a model, $\tau_{\text {eng, }, 0}$, but aging and piece-to-piece variability can render this nominal model inaccurate. Assuming that the uncertainty can be adequately captured by an offset and a multiplier, so that $\tau_{\text {eng }}=\theta_{1} \cdot \tau_{\text {eng, } 0}+$ $\theta_{2}$, and by defining

$$
\begin{gathered}
x=\omega, \quad u=-\frac{1}{J} \tau_{\text {load }}, \quad z_{1}=\frac{1}{J} \tau_{\text {eng }, 0}, \quad z_{2}=\frac{1}{J} \cdot 1 \\
\theta=\left[\begin{array}{l}
\theta_{1} \\
\theta_{2}
\end{array}\right], \quad z=\left[\begin{array}{l}
z_{1} \\
z_{2}
\end{array}\right]
\end{gathered}
$$




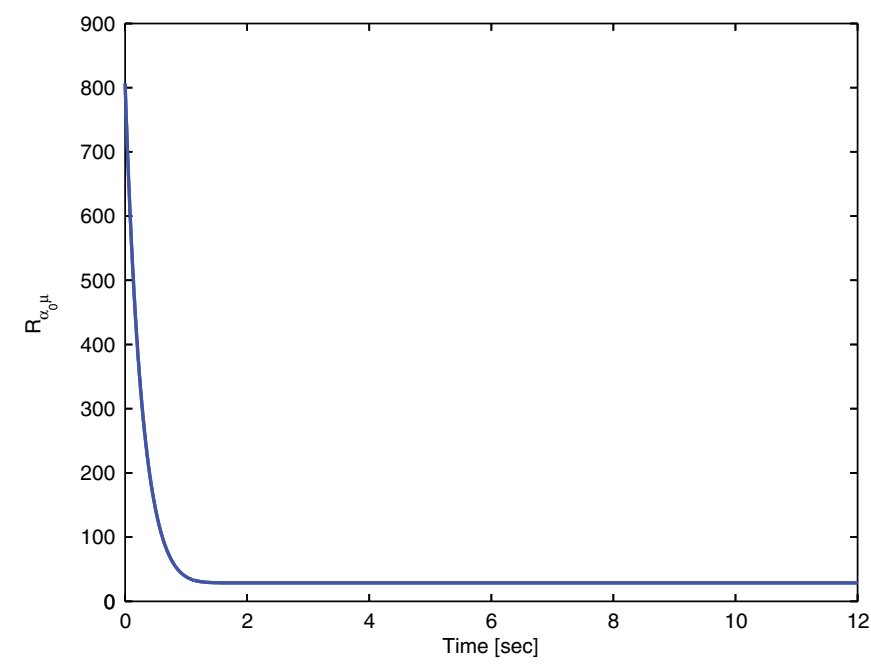

Figure 4. Time history of $R_{\alpha_{0} \mu}(t)$.
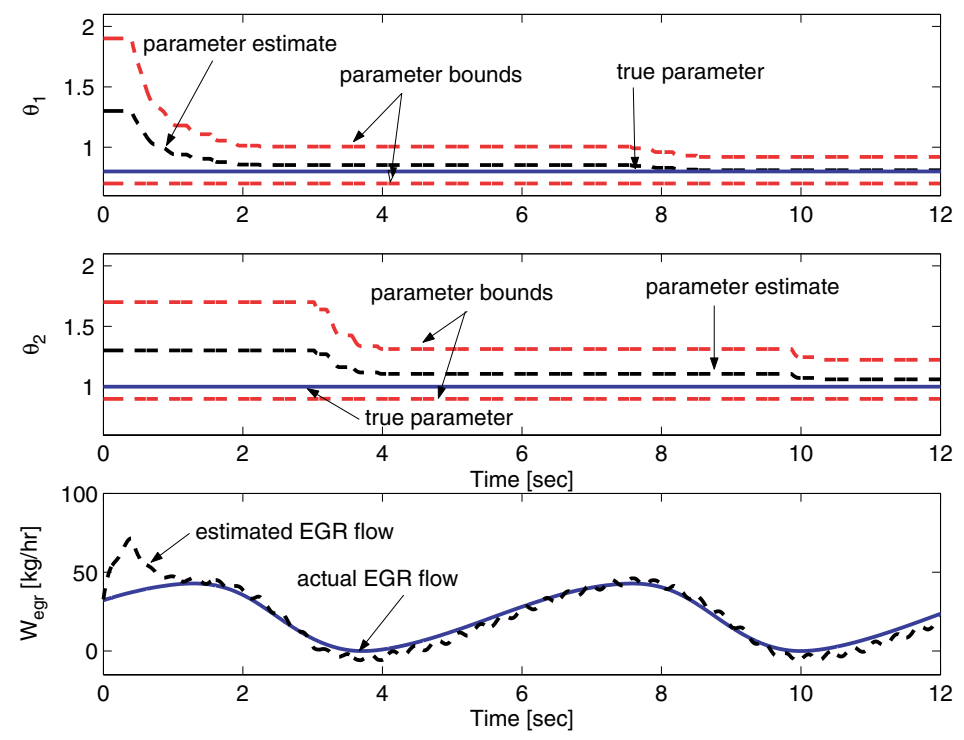

Figure 5. Time histories (from (a) at the top to (c) at the bottom) of: (a) true parameter $\theta_{1}$ (solid), parameter estimate $\hat{\theta}_{1}=c_{1}$ (dashed), parameter bounds $c_{1}+l_{1}$ and $c_{1}-l_{1}$ (dashed); (b) true parameter $\theta_{2}$ (solid), parameter estimate $\hat{\theta}_{2}=c_{2}$ (dashed), parameter bounds $c_{2}+l_{2}$ and $c_{2}-l_{2}$ (dashed); and (c) actual EGR flow $W_{\text {egr }}$ (solid) and estimated EGR flow, $\hat{W}_{\text {egr }}$ (dashed).

the system is transformed into the standard form (1). The accurate knowledge of $\tau_{\text {eng }}$ is required for engine and powertrain control, in particular, for drivability and engine/transmission coordination during gear shifts. Additionally, the estimate of $\tau_{\text {load }}$ can be included in the feedforward compensation to improve idle speed or cruise control. Experiments are currently being pursued for this application that will be reported elsewhere. 


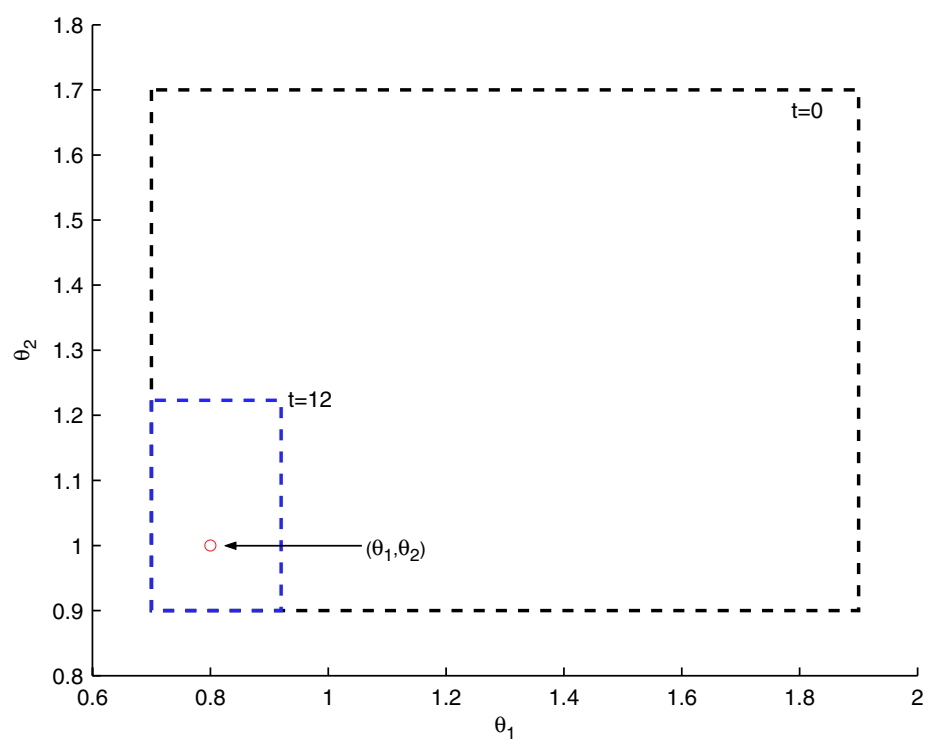

Figure 6. The rectangles $P(0)$ and $P(k)$ at $t=k \Delta=12 \mathrm{~s}$.

\section{CONCLUDING REMARKS}

In this paper, we addressed a problem of simultaneously estimating an input and an unknown parameter in a dynamic system. This problem is not solvable without special assumptions. We showed that this problem can be treated by combining a conventional input observer with a setmembership algorithm for parameter estimation. The set-membership algorithm provides a set of plausible parameter estimates consistent with the a priori available information including bounds on the unknown input and expected input observer errors for the case with no parametric uncertainty. The potential applications to engine control have been discussed and illustrated with a simulation example.

\section{APPENDIX A: GENERALIZATION TO HIGHER ORDER SYSTEMS}

The theoretical developments in this paper mainly concern a first-order system which was relevant to a particular class of powertrain control applications. We now discuss how certain classes of higher order systems can be treated.

Suppose a higher order system can be transformed into the form,

$$
\begin{aligned}
& \dot{x}=Z^{\mathrm{T}}(t) \theta+u(t) \\
& y(t)=x(t)+\zeta(t)
\end{aligned}
$$

where $x$ is an $n$-vector state, $Z(t)$ is an $m \times n$ matrix, $\theta$ is an $m$-vector of constant unknown parameters, $u$ is an $n$-vector unknown input, $y$ is an $n$-vector measured output, and $\zeta$ is an 
$n$-vector noise. In this case, $\mu, \underline{u}(t), \bar{u}(t)$ and $h$ are $n$-vectors, and (2), (3), (4) are understood to apply to each vector component.

System (A1) can be equivalently viewed as a system of $n$ differential equations of the form,

$$
\begin{aligned}
& \dot{x}_{1}=Z_{11}(t) \theta_{1}+Z_{21}(t) \theta_{2}+\cdots+Z_{m 1}(t) \theta_{m}+u_{1}(t) \\
& \dot{x}_{2}=Z_{12}(t) \theta_{1}+Z_{22}(t) \theta_{2}+\cdots+Z_{m 2}(t) \theta_{m}+u_{2}(t) \\
& \vdots \\
& \dot{x}_{n}=Z_{1 n}(t) \theta_{1}+Z_{2 n}(t) \theta_{2}+\cdots+Z_{m n}(t) \theta_{m}+u_{n}(t)
\end{aligned}
$$

where $Z_{i j}$ is the $(i, j)$ th entry of the matrix $Z$. Each of these differential equations is in the standard form (1) and can be used as a basis for generating a set-membership estimate (13), i.e. $\hat{\theta} \in F_{i}(t)$, at time $t$. Combining the information for $i=1, \ldots, n$, we obtain $\hat{\theta} \in \bigcap_{i=1, \ldots, n} F_{i}(t)$.

As a specific example of the transformation into form (A1), consider a general nonlinear system,

$$
\dot{\chi}=f(\chi)+g(\chi) v+p(\chi) \theta
$$

where $\chi(t) \in \mathbf{R}^{q}$ is the state, $v(t) \in \mathbf{R}^{n}$ is the unknown input, and $\theta \in \Theta^{0} \subset \mathbf{R}^{m}$ is the vector of unknown parameters. The objective is to simultaneously estimate $v(t)$ and $\theta$. Assuming that the state, $\chi$, can be measured, let $x(t) \in \mathbf{R}^{n}$ be some output of the system so that,

$$
x=h(\chi)
$$

Note that the dimensionality of $x$ is the same as that of $v$. Then,

$$
\dot{x}=\frac{\partial h}{\partial \chi}(f(\chi)+p(\chi) \theta+g(\chi) v)
$$

There are two approaches for converting (A3) into form (A1). In the first approach, we define

$$
Z=\left(\frac{\partial h}{\partial \chi} p(\chi)\right)^{\mathrm{T}}, \quad u=\frac{\partial h}{\partial \chi}(f(\chi)+g(\chi) v)
$$

and obtain a system of form (A1). If the $n \times n$ matrix $((\partial h / \partial \chi) g(\chi(t)))$ is invertible then the estimate of $v(t), \hat{v}(t)$, can be uniquely computed from the estimate of $u(t), \hat{u}(t)$, as $\hat{v}(t)=((\partial h / \partial \chi) g(\chi(t)))^{-1}(\hat{u}(t)-(\partial h / \partial \chi) f(\chi(t)))$. In the second approach to estimating $v(t)$ and $\theta$, we view the term, $(\partial h / \partial \chi) f(\chi)$ in $(\mathrm{A} 3)$, as $(\partial h / \partial \chi) f(\chi) \cdot \bar{\theta}$, where $\bar{\theta}=1$ is an additional constant parameter to be estimated along with $\theta$. In that case, to convert (A3) to form (A1) we can define $u=((\partial h / \partial \chi) g(\chi)) v$,

$$
Z=\left[\begin{array}{l}
\left(\frac{\partial h}{\partial \chi} p(\chi)\right)^{\mathrm{T}} \\
\left(\frac{\partial h}{\partial \chi} f(\chi)\right)^{\mathrm{T}}
\end{array}\right], \quad \tilde{\theta}=\left[\begin{array}{c}
\theta \\
\bar{\theta}
\end{array}\right]
$$

and impose (5) in the form $\tilde{\theta} \in \tilde{\Theta}^{0}=\Theta^{0} \times\{1\}$. If the $n \times n$ matrix $((\partial h / \partial \chi) g(\chi(t)))$ is invertible, then the estimate of $v(t), \hat{v}(t)$, can be uniquely computed from the estimate of $u(t), \hat{u}(t)$, as $\hat{v}(t)=((\partial h / \partial \chi) g(\chi(t)))^{-1} \hat{u}(t)$.

To provide a brief illustration of a practical problem which can be treated with these ideas, suppose in the flow estimation problem treated in Section 6, the air flow sensor is slow and its 


\section{SIMULTANEOUS INPUT AND PARAMETER ESTIMATION}

dynamics are

$$
\dot{W}_{\mathrm{th}, m}+a W_{\mathrm{th}, m}=a W_{\mathrm{th}}
$$

where $W_{\mathrm{th}, m}$ is the air flow sensor measurement, $W_{\mathrm{th}}$ is the actual flow of the air through the throttle and $1 / a$ is the air flow meter time constant. In this case, one may not use $W_{\text {th, } m}$ in place of $W_{\text {th }}$ in (23) when estimating $W_{\text {egr }}$ and unknown parameters. Suppose, however, that a nominal model for the throttle flow, $W_{\mathrm{th}, 0}$, is available. For instance, such a nominal model can be based on an orifice equation (see Reference [19]), and it determines the throttle flow as a function of pressure drop across the throttle and throttle position. To compensate for aging and piece-to-piece variability, an adaptive model for the throttle flow, $W_{\mathrm{th}}$, can be introduced as follows:

$$
W_{\mathrm{th}}=\theta_{2} W_{\mathrm{th}, 0}+\theta_{3}
$$

where $\theta_{2}$ and $\theta_{3}$ are the offset and the multiplier modifiers, respectively. The parameterization of the cylinder flow is as in Section 6, i.e. $W_{\text {cyl }}=\theta_{1} W_{\text {cyl }, 0}$. Then the problem of simultaneously estimating $\theta_{1}, \theta_{2}, \theta_{3}$ and $W_{\text {egr }}$ on the basis of (23) and (A4) can be solved using the higher order case approach described in this appendix.

\section{APPENDIX B: A SET-MEMBERSHIP COMPUTATIONAL ALGORITHM}

In our approach, we replace the updates of $F_{d}(k)$ with the updates of an $m$-dimensional rectangle, $P(k)$, which overbounds $F_{d}(k)$, i.e. $F_{d}(k) \subset P(k)$. The centre of $P(k)$ is denoted by $c(k) \in \mathbf{R}^{m}$ and its half-sides are $l_{1}(k), \ldots, l_{m}(k)$, i.e.

$$
P(k)=\left\{\theta=\left[\theta_{1}, \ldots, \theta_{m}\right]^{\mathrm{T}} \in \mathbf{R}^{m}: c_{i}(k)-l_{i}(k) \leqslant \theta_{i} \leqslant c_{i}(k)+l_{i}(k), \quad i=1, \ldots, m\right\}
$$

Note that the Chebyshev centre of $P(k)$ is $c(k)$ and thus $\hat{\theta}(t)=c(k)$ for $k \cdot \Delta \leqslant t<(k+1) \cdot \Delta$.

A new rectangle, $P(k)$, is formed recursively so that $P(k-1) \bigcap \Theta(k \Delta) \subset P(k)$. The computationally straightforward procedure to update $c(k)$ and $l(k)$ amounts to tightening each side of the rectangle $P(k-1)$ to overbound the set $P(k-1) \bigcap \Theta(k \Delta)$.

Referring to (12), note first that $\Theta(k \Delta)=\left\{\theta \in \mathbf{R}^{m}: s(k) \theta+r_{1}(k) \leqslant 0,-s(k) \theta+r_{2}(k) \leqslant 0\right\}$, where

$$
\begin{gathered}
s(k)=\phi^{\mathrm{T}}(k \Delta) \\
r_{1}(k)=-\bar{u}(k \Delta)-R_{\alpha_{0} \mu}(k \Delta)+\varepsilon(k \Delta)+\alpha_{0} y(k \Delta) \\
r_{2}(k)=\underline{u}(k \Delta)-R_{\alpha_{0} \mu}(k \Delta)-\varepsilon(k \Delta)-\alpha_{0} y(k \Delta)
\end{gathered}
$$

Therefore, $\Theta(k \Delta)$ is the set 'in-between' two parallel hyper-planes $H_{1}(k)=\left\{\theta \in \mathbf{R}^{m}\right.$ : $\left.s(k) \theta+r_{1}(k)=0\right\}$ and $H_{2}(k)=\left\{\theta \in \mathbf{R}^{m}: s(k) \theta-r_{2}(k)=0\right\}$. To determine whether an $(m-1)$-dimensional face of the rectangle can be tightened, we determine all intersections of $H_{1}$ and $H_{2}$ with the extended edges of the rectangle perpendicular to that face. The extended edges are straight lines that pass through the edges. A face can be tightened if these intersection points, which are explicitly and easily computable, and the opposite face lie in the same halfspace. Figure A1 illustrates the procedure in the case $m=2$. Note that the result is independent of the order in which the sides are tightened. 


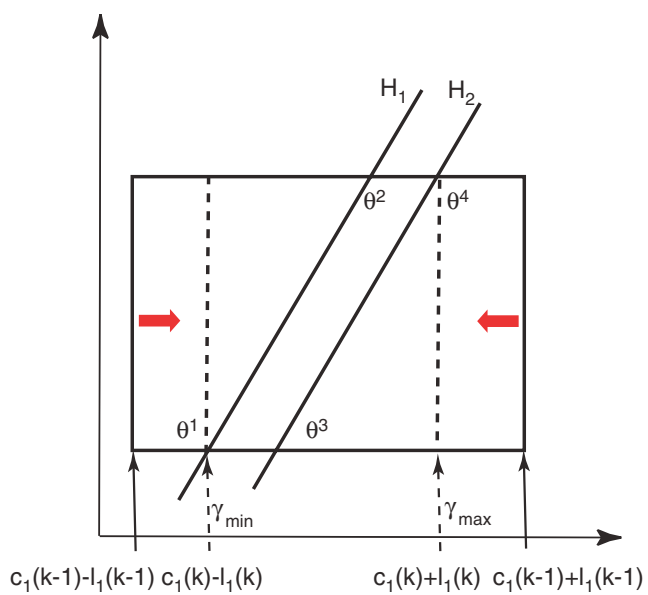

(a)

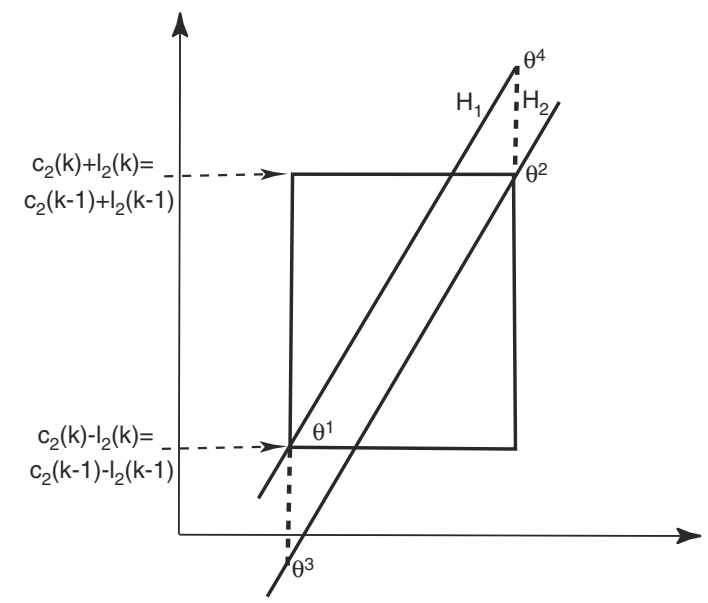

(b)

Figure A1. Algorithm for updating $P(k-1)$ to $P(k)$ for $m=2$ : (a) update of $c_{1}(k-1), l_{1}(k-1)$ to $c_{1}(k), l_{1}(k)$, both vertical faces are tightened; and (b) update of $c_{2}(k-1), l_{2}(k-1)$ to $c_{2}(k), l_{2}(k)$, the horizontal faces cannot be tightened.

This algorithm for updating $c(k-1)$ and $l(k-1)$ to, respectively, $c(k)$ and $l(k)$ is now explicitly specified.

Algorithm for updating $P(k-1)$ to $P(k), k>0$

Step 1: If $s(k) \neq 0$ proceed to Step 2. If $s(k)=0, r_{1}(k) \leqslant 0, r_{2}(k) \leqslant 0$ assign $c(k):=c(k-1)$, $l(k):=l(k-1)$, Exit. If $s(k)=0$ and either $r_{1}(k)>0$ or $r_{2}(k)>0$, then $P(k)=\emptyset$, Exit and follow the Reset procedure.

Step 2: Set $i=0, c(k):=c(k-1), l(k):=l(k-1)$.

Step 3: Set $i=i+1$.

Step 4: If $s_{i}(k)=0$ then Goto Step 3. If $s_{i}(k) \neq 0$, compute all intersection points, $\theta_{i}^{q}$, $q=1, \ldots, 2^{m}$, of $H_{1}$ and $H_{2}$ with the extended edges parallel to the $i$ th ort in $\mathbf{R}^{m}$ :

$$
\begin{gathered}
\theta_{i}^{q}=\frac{1}{s_{i}(k)}\left(-s_{1}(k)\left(c_{1}(k) \pm l_{1}(k)\right)-\cdots-s_{i-1}(k)\left(c_{i-1}(k) \pm l_{i-1}(k)\right)\right. \\
-s_{i+1}(k)\left(c_{i+1}(k-1) \pm l_{i+1}(k-1)\right)-\cdots \\
\left.-s_{m}(k)\left(c_{m}(k-1) \pm l_{m}(k-1)\right)-r_{1}(k)\right) \\
\theta_{i}^{q}=\frac{1}{s_{i}(k)}\left(-s_{1}(k)\left(c_{1}(k) \pm l_{1}(k)\right)-\cdots-s_{i-1}(k)\left(c_{i-1}(k) \pm l_{i-1}(k)\right)\right. \\
\left.-s_{i+1}(k)\left(c_{i+1}(k-1) \pm l_{i+1}(k-1)\right)-\cdots-s_{m}(k)\left(c_{m}(k-1) \pm l_{m}(k-1)\right)+r_{2}(k)\right)
\end{gathered}
$$

Step 5: Set

$$
\begin{aligned}
& \gamma_{\max }=\min \left\{\max \left\{\theta_{i}^{q}, q=1, \ldots, 2^{m}\right\}, c_{i}(k-1)+l_{i}(k-1)\right\} \\
& \gamma_{\min }=\max \left\{\min \left\{\theta_{i}^{q}, q=1, \ldots, 2^{m}\right\}, c_{i}(k-1)-l_{i}(k-1)\right\}
\end{aligned}
$$




\section{SIMULTANEOUS INPUT AND PARAMETER ESTIMATION}

and

$$
\begin{aligned}
& c_{i}(k)=\frac{1}{2}\left(\gamma_{\max }+\gamma_{\min }\right) \\
& l_{i}(k)=\frac{1}{2}\left(\gamma_{\max }-\gamma_{\min }\right)
\end{aligned}
$$

Step 6: If $l_{i}(k)<0$, then $P(k)=\emptyset$, Exit and follow the Reset procedure. Otherwise, Goto Step 7.

Step 7: While $i<m$, Goto Step 3. Otherwise, Exit.

The algorithm can be initialized with $c(0)$ and $l(0)$ for which the corresponding $P(0)$ in $(\mathrm{B} 1)$ overbounds $\Theta^{0}$, i.e. $\Theta^{0} \subset P(0)$. For $m=1$, a different, even simpler to implement algorithm for updating $\hat{\theta}(t)$ has been reported in Reference [2]. That algorithm, however, tends to keep $\hat{\theta}(t)$ at or near the boundary of $F_{d}(k)$ and not close to its Chebyshev centre.

The Reset procedure is used if $P(k)=\emptyset$ for some $k$. This cannot happen under the ideal assumptions, but it may happen in the real application if the assumptions are violated, for example, as a result of an abrupt change in the unknown parameter $\theta$ or due to the bounds not being satisfied. To account for possible abrupt parameter changes, the Reset procedure assigns $c(k)=c(k-1), l(k)=l(0)$ thereby reconstituting the rectangle $P(k)$ to its original size and centring it around the latest valid parameter estimate, $c(k-1)$. Strategies can also be devised to revise the bounds $\mu, h, \underline{u}$ and $\bar{u}$ on-line if $P(k)=\emptyset$ tends to occur frequently.

For the case $m=1$, an alternative and even easier to implement parameter estimation procedure has been proposed in Reference [2]. In Reference [2], if $s(k) \neq 0$ then the update law for the parameter estimate, $\hat{\theta}(k \Delta)$, takes the following form:

$$
\hat{\theta}(k \Delta)= \begin{cases}\frac{r_{2}(k)}{s(k)} & \text { if } s(k) \hat{\theta}((k-1) \Delta)<r_{2}(k) \\ \frac{-r_{1}(k)}{s(k)} & \text { if } s(k) \hat{\theta}((k-1) \Delta)>-r_{1}(k) \\ \hat{\theta}((k-1) \Delta) & \text { if } r_{2}(k) \leqslant s(k) \hat{\theta}((k-1) \Delta) \leqslant-r_{1}(k)\end{cases}
$$

If $s(k)=0$ then $\hat{\theta}(k \Delta)=\hat{\theta}((k-1) \Delta)$, since, under our assumptions, $-r_{1}(k) \geqslant r_{2}(k)$. This procedure guarantees that $\hat{\theta}(k \Delta) \in F_{d}(k \Delta)$, although, in general, $\hat{\theta}(k \Delta)$ is not the Chebyshev centre of $F_{d}(k \Delta)$.

\section{REFERENCES}

1. Belforte G, Tay TT. Two new algorithms for linear models with unknown but bounded measurement noise. IEEE Transactions on Automatic Control 1993; 38(8):1273-1279.

2. Kolmanovsky I, Siverguina I. Adaptive identification schemes in presence of bounded disturbances: an automotive case study. Proceedings of IEEE Conference on Decision and Control, Orlando, FL, 2001; 508-513.

3. Kolmanovsky I, Sivergina I, Sun J. Combined input and parameter estimation with input observers and set membership parameter bounding. Proceedings of IEEE Conference on Decision and Control, Atlantis, Paradise Island, Bahamas, 2004; 5192-5197.

4. Bartolini C, Ferrara A, Stotsky A. Robustness and performance of an indirect adaptive control scheme in presence of bounded disturbances. IEEE Transactions on Automatic Control 1999; 44(4):789-793.

5. Bertsekas DP, Rhodes IB. Recursive state estimation for a set-membership description of uncertainty. IEEE Transactions on Automatic Control 1971; 16(2):117-128. 


\section{KOLMANOVSKY, I. SIVERGINA AND J. SUN}

6. Schweppe FC. Recursive state estimation: unknown but bounded errors and system inputs. IEEE Transactions on Automatic Control 1968; 13:22-28.

7. Schweppe FC. Uncertain Dynamic Systems. Prentice-Hall: Englewood Cliffs, NJ, U.S.A.

8. Bai EW, Huang YF. Variable gain parameter estimation algorithms for fast tracking and smooth steady-state. Automatica 2000; 36:1001-1008.

9. Basile G, Marro G. On the observability of linear, time-invariant systems with unknown inputs. Journal of Optimization Theory and Applications 1969; 3:410-415.

10. Sivergina IF. Invertibility and observability of evolution systems. Doklody Akademii Nauk 1996; 351:304-308 (in Russian).

11. de Mathelin M, Lozano R. Robust adaptive identification of slowly time-varying parameters with bounded disturbances. Automatica 1999; 35:1291-1305.

12. Vahidi A, Druzhinina M, Stefanopoulou A, Peng H. Simultaneous mass and time-varying grade estimation for heavy-duty vehicles. Proceedings of 2003 American Control Conference, Denver, CO, 2003; 4951-4956.

13. Stotsky A. Lyapunov design for convergence rate improvements in adaptive control. International Journal of Control 1993; 57(2):501-504.

14. Bai EW, Ye Y, Tempo R. Bounded error parameter estimation: a sequential analytic center approach. IEEE Transactions on Automatic Control 1999; 44(6):1107-1117.

15. Fogel E, Huang F. On the value of information in system identification-bounded noise case. Automatica 1982; 18:229-238.

16. Chisci L, Garulli A, Zappa G. Recursive state bounding by parallelotopes. Automatica 1996; 32(7):1049-1055.

17. Stotsky A, Kolmanovsky I. Application of input estimation techniques to charge estimation in automotive engines. IFAC Journal of Control Engineering Practice 2002; 10(12):1371-1383.

18. Kolmanovsky I, Sun J, Druzhinina M, van Nieuwstadt M. Charge control for direct injection engines with EGR. Proceedings of American Control Conference, Chicago, IL, 2000; 34-38.

19. Heywood JB. Internal Combustion Engine Fundamentals. McGraw-Hill, Inc., 1988. 